Faculdade

de Ciências Econômicas UFRGS
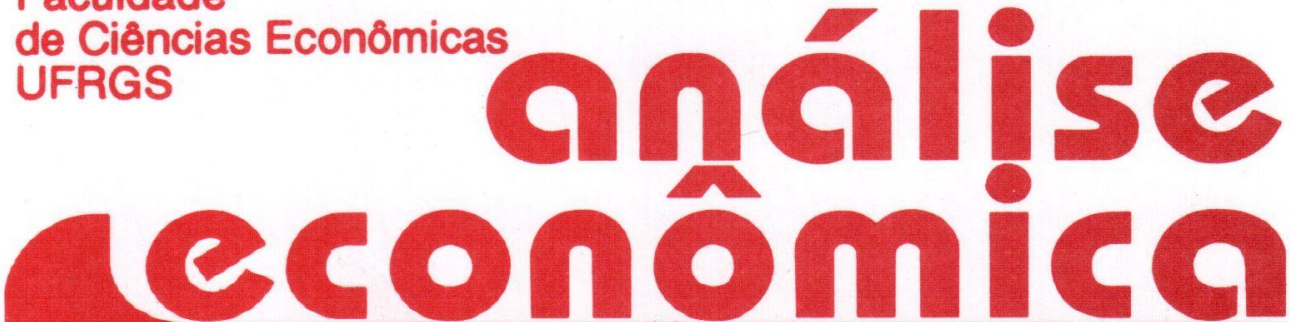

- EQUILIBRIO, PROGRESSO TÉCNICO E DESIGUALDADES REGIONAIS

Carlos Roberto Azzoni

- DESENVOLVIMENTO POLARIZADO E DESEQUILÍBRIOS REGIONAIS Nali de Jesus de Souza

- OS NOVOS CLÁSSICOS E O MÉTODO Carlos Magno Lopes

- ECONOMLAS DE MERCADO E DEMANDA EFETIVA Gilberto Tadeu Lima

- MEASURES OF CAPACITY UTILIZATION Marcelo S. Portugal

- ASPECTOS DO CONTROLE EM UM MODELO DINÂMICO

Marat Rafikow

Pedro Augusto P. Borges

- A FIRMA EM UM AMBIENTE INFLACIONÁRIO

Carmen A.do V.C. Feijó

- CUSTOS E BENEFICIOS DA INTEGRAÇĀO REGIONAL Marco Antônio Montoya

- A REESTRUTURAÇÃO DA ECONOMIA MUNDIAL Hoyêdo Nunes Lins

- O MERCADO COMO PROCESSO: A ABORDAGEM AUSTRÍACA

Fernando Caputo Zanella

- GARY BECKER: PRÊMIO NOBEL DE ECONOMIA DE 1992

Giácomo Babinotto Neto

LIVROS RECEBIDOS

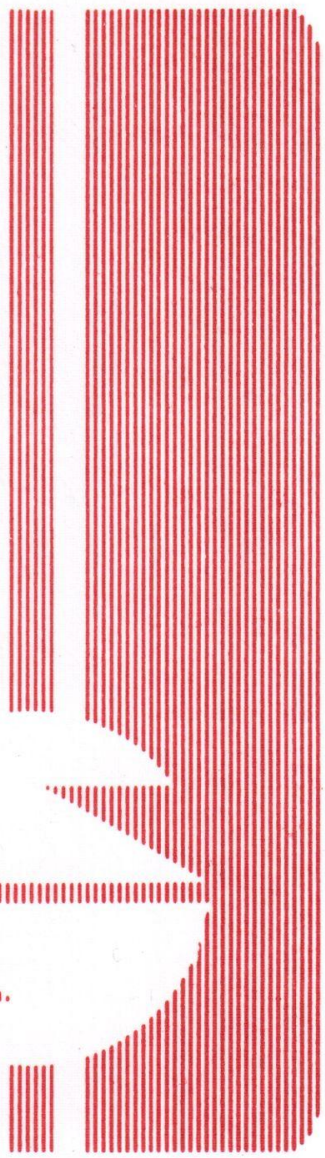


UNIVERSIDADE FEDERAL DO RIO GRANDE DO SUL Reitor: Prof. Hélgio Henrique Casses Trindade

FACULDADE DE CIENCIAS ECONOMICAS

Diretor: Prof. Pedro Cézar Dutra Fonseça

CENTRO DE ESTUDOS E PESQUISAS ECONOMICAS

Diretor: Prof. Roberto Pires Pacheco

DEPARTAMENTO DE CIENCIAS ECONÓMICAS

Chefe: Prof. Fernando Ferrari Filho

CURSO DE POS-GRADUAÇAOO EM ECONOMIA

Coordenador: Prof. Joăo Rogério Sanson

CURSO DE POS-GRADUAÇÂO EM ECONOMIA RURAL

Coordenador: Prof. Juvir Luiz Mattuella

CONSELHO EDITORIAL: Achyles Barcelos da Costa, Aray Miguel Feldens, Atos Freitas Grawunder, Carlos Augusto Crusius, Ernani Hickmann, Fernando Ferrari Filho, Joāo Rogério Sanson, Juvir Luiz Mattuella, Marcelo Savino Portunal, Maria Imilda da Costa e Silva, Nali de Jesus de Souza, Nuno Renan Lopes de Figueiredo Pinto, Otília Beatriz Kroeff Carrion, Otto Guilherme Konzen, Paulo Alexandre Sphor, Pedro Cezar Dutra Fonseca, Reinaldo I gnacio Adams, Roberto Camps Moraes, Valter José Stülp, Yeda Rorato Crusius, David Garlow (Wharton Econometrics Forecasts Association, E.U.A.), Edgar Augusto Lanzer (UFSC), Eleutério F. S. Prado (USP), Fernando Holanda Barbosa (FGV/RJ), Gustavo Franco (PUC/RJ), Joaquim Pinto de Andrade (UnB), Juan H. Moldau (USP), Werner Baer (Univ. de lllinois, E.U.A.).

COMISSĀO EDITORIAL: Atos Freitas Grawunder, Pedro Cezar Dutra Fonseca, Reinaldo Ignacio Adams e Roberto Camps Moraes.

EDITOR: Prof. Nali de Jesus de Souza

SECRETARIA: Maria Ivone de Mello (normalizaçāo), Vanete Ricacheski (revisão de textos).

FUNDADOR: Prof. Antônio Carlos Santos Rosa

Os materiais publicados na revisța Análise Econômica sāo da exclusiva responsabilidade dos autores. E permitida a reprodıçāo total ou parcial dos trabalhos, desde que seja citada a fonte.

Aceita-se permuta com revistas congêneres. Aceitam-se, também, livros para divulgaçāo, elaboraçāo de resenhas ou recensōes.

Toda correspondência, material para publicação (vide normas na terceira capa), assinaturas e permutas devem ser dirigidos ao seguinte destinatário:

\author{
PROF. ROBERTO CAMPS MORAES \\ Revista Análise Económica \\ Av. Joāo Pessoa, 52 \\ CEP 90040-000 - PORTO ALEGRE (RS), BRASIL \\ Telefones: (051) 228-1633, ramal 3440
}

Fax: (051) 225-1067 


\title{
OS NOVOS CLÁSSICOS E O MÉTODO
}

\author{
Carlos Magno Lopes*
}

\section{SINOPSE}

No início dos anos setenta, os Novos Clássicos iniciaram um processo de análise crítica do pensamento keynesiano, o qual destacava, primordialmente, a ausência de fundamentos microeconômicos na General Theory. Como alternativa ao keynesianismo, formularam uma proposta que consiueravam revolucionária: a construçäo de uma teoria de ciclos que pudesse ser expressa na forma de equilfbrio competitivo. Neste sentido propuseram leis gerais sobre o comportamento cíclico da economia a partir da identificaçāo de co-movements. A partir daí, introduziram um modelo estocástico e dinâmico, mas que assume a realidade observável como sendo estacionária, repetitiva:"Se nāo for este o caso, o modelo torna-se dinamicamente instável e incapaz de fazer previsöes.

\section{INTRODUÇÃO}

A importância dos ciclos econômicos na agenda de pesquisa dos economistas tem variado ao longo do tempo em função direta da instabilidade experimentada pela economia mundial. Nos tempos atuais, parece haver um renovado esforço de melhor entender as razões que levam à persistente existência de ciclos. Isto reflete, em larga extensão, a crescente insatisfação com as explicaçōes "keynesianas" e monetaristas ante os erráticos movimentos de preços, do produto e do emprego, notadamente na década de setenta. Neste contexto histórico, um relativamente obscuro artigo de Muth (1961) que estabelecia como critério de racionalidade a utilização ótima de todas as informações disroníveis, por parte dos agentes econômicos no processo de formação de expectativas, foi resgatado por Lucas e Rapping (1969), dando início à construção de um paradigma cujos proponentes viriam a ser conhecidos como Novos Clássicos.

O estudo de ciclos econômicos, como se sabe, é de extraordinária complexidade, pois superpõe-se às teorias de crescimento, do progresso técnico, da distribuição, da moeda, da inflação e das expectativas, além, é claro, aos arranjos institucionais orgânicos intrínsecos aos processos econômicos. Assim sendo, o

* Stanford University (Visiting Scholar) e Universidade Federal do Ceara, Professor do Curso de Mestrado em Economia (CAEN). Agradeço a Joaquim Andrade e aos participantes do seminário de macroeconomia em Stanford pelas sugestöes. Esta pesquisa foi parcialmente financiada pelo CNPq.

$$
\begin{array}{c|c}
\hline \begin{array}{c}
\text { Cód. AEA } \\
030
\end{array} & \text { Palavras-Chave: } \\
& \text { Ciclos, dinâmica e equilíbrio }
\end{array}
$$


estudo do método ${ }^{1}$ que orienta a construção dos diversos paradigmas assume excepcional importância. ${ }^{2}$ Felizmente, no caso dos Novos Clássicos, é possível ir-se além de inferências sobre o método, conquanto a explicitação deste tem merecido significativa atenção por parte dos teóricos desta Escola.

Este artigo objetiva, portanto, identificar e analisar os fundamentos centrais do método comum aos Novos Clássicos, sem atribuir especial relevância às particularidades das várias vertentes, ${ }^{3}$ situando este paradigma no seu contexto de formaçấo histórica. Não é objetivo deste artigo fazer uma avaliação teórica ou empírica deste paradigma.

\section{ANTECEDENTES E MOTIVAÇÕES}

Para melhor entender a gênesis do processo que engendrou a construção do paradigma ora em análise, faz-se necessário uma breve digressão sobre a evolução da agenda macroeconômica nos anos sessenta e setenta, bem como da aparente ruptura com os modelos de inspiração keynesiana, que exerceram uma imperturbável hegemonia no período de Pos-Guerra. Curiosamente, a contribuição seminal de Lucas e Rapping (1969), hoje vista como o início de um ataque frontal à tradição keynesiana era percebida como uma tentativa de prover o keynesianismo com fundamentos microeconômicos. Lucas (1981, p.2) destaca que: "Our objective at the time, as is clear enough from the introduction of paper and such defensive efforts as note 3 , was not to challenge the current, Keynesian orthodoxy but rather to contribute to it by constructing a 'micro-economic foundation' for the wage-price sector of macroeconometric models". Este objetivo, no entanto, que procurava determinar o critério de racionalização das firmas e das famílias nas decisões de emprego, esbarrava na suposta rigidez do método de Keynes. Segundo Lucas (ibid. p.3):

Evidently, this could not be carried out at the level of the static, one -period indiference diagram of the sort we ise to explain, for example, the secular decline in hours worker per worker per week (This is chapter 2 of the General Theory).

Um dos desafios, portanto, seria o de encontrar uma solução para evitar este problema, ou seja, romper com o caráter supostamente estático do método de Keynes. Lucas (1981, p.3) argumenta que, em Lucas e Rapping (1961), "we adopted a two-period set-up in which expected future prices, in addition to current prices, could play a role". A abordagem assim se tornaria dinâmica, a partir da

1 A definição de método aqui adotada é a de Hicks (1985, p. 1), para quem método é "...a family, or class, of models". Modelo é definido como "... a piece of theory, a theoretical construction, which is in tended to be applied to a certain range of facts". Neste artigo, o método é forma; metodologia é estudo dos métodos no sentido filosófico-científico.

2 . Neste sentido, Friedman (1953) e Hicks (1980-81) servem como exemplos, entre outros, de autores que julgaram fazer uma exposição explícita dos métodos por eles empregados.

3 Nos anos oitenta, por exemplo, a corrente da Teoria dos Ciclos Reais parece ter-se tornado a mais fluente entre os Novos Clássicos. Além disso, Lucas, Sargent e outros exploraram, neste mesmo período, princípios téricos distintos áaqueles do período forma tivo, mantendo, con tudo, o método. 
introdução de interações intertemporais. Outro obstáculo de excepcional importância, no entanto, teria de ser removido, isto é, a contradição de expressar a Teoria dos Ciclos em termos de equilibrio geral competitivo. Mais uma vez, os Novos Clássicos encontraram uma engenhosa solução:

The positive rationale of the "classical restoration" is that the contradiction between the static general economic equilibrium and the dynamic cmpirical econom ic cycle can be overcome by applying the concept of abstraction of which phenomena are seen as manifestation. In other words it is assumed that general economic equilibrium refers to the sthochastic process of which observed phenomena constitute just one possible realization. (Vercelli, 1991, p.129)

Lucas considerou esta estratégia como de difícil aceitação, possivelmente sujeita a controvérsias. Sobre Lucas e Rapping (1969), Lucas (1981, p.3) destaca que "We were aware, of course, that working toward a competitive-equilibrium account of the cyclical behavior of employment would be viewed in some circles as quixotic or even subversive, but we supposed that the basis for this widespread view was no deeper than the belief that such an account was not possible to device". Lucas acreditou estar fazendo uma revolução!

\section{ECONOMIA COMO CIÊNCIA: OS FUNDAMEN'TOS DO MÉTODO}

Na seção anterior, procurou-se mostrar que a superação do método de Keynes teria sido possível, segundo os Novos Clássicos, a partir da introdução do equilibrium method, uma extensão para a macroeconomia da abordagem do equilíbrio geral walrasiano. Como será discutido adiante, o equilibrium method apoia-se na noção de equilíbrio estacionário em processos estocásticos. A aplicação deste conceito é claramente justificada pela visão, comum aos Novos Clássicos, sobre a Ciência Econômica. Lucas $(1981$, p.272) argumenta que "...a theory is not a collection of assertions about the behavior of the actual economy but rather an explicit set of instructions for building a parallel of analogue system, a mechanical, imitation economy". Lucas acredita que o grau de realismo de um modelo econômico é irrelevante: o importante é a utilidade do mesmo para ajudar a fazer previsões sobre o comportamento da economia. Alem disso, Lucas (1981, p.271) sustenta que "Any model that is well enough articulated to give clear answers to the questions we put it will necessarily be artificial,abstract, patently "unreal"". A noção de realidade para Lucas, contudo, possui um significado particular. Entender a "realidade" significa extrair de séries temporais regras comportamentais que estabeleçam como indivíduos que agem inteligentemente (segundo o princípio da otimização) reagem a mudanças bem definidas na economia. Isto só seria possível através da utilização sistemática de técnicas econométricas. Lucas (ibid., p.288) não poderia ser mais claro quanto a este fato: "Our task as I see it... is to write a Fortran program that will accept specific economic policy rules as 'input'and will generate as 'output' statistics describing the operating characteristics of time series we care about, which are predicted to result from these policies". 
Neste ponto, alguns resultados importantes emergem. Em primeiro lugar, a teoria incapaz de fazer previsões não passa pelo critério de "utilidade", devendo, portanto, ser descartada. Além disso, a teoria construída por "intiospecção", isto é, não derivada diretamente de estudos empíricos sistemáticos, representaria um desvio do método científico. A teoria econômica para ser científica, segundo os Novos Clássicos, deve resultar de rigorosa análise de séries temporais, que identifique regularidades no comportamento de variáveis relevantes ao longo dos ciclos, a partir das quais relações causais possam ser estabelecidas. ${ }^{4}$ Além disso, como dito anteriormente, a teoria macroeconômica só tem sentido se fundamentada em relações microeconômicas.

\section{TEORIA: CONSTRUÇÃO E MÉTODO}

O objeto central de estudo dos Novos Clássicos, os ciclos econômicos, ilustra com riqueza de detalhes a utilização do método empregado por estes autores. Desta forma, será desenvolvido abaixo um esforço de associar os buildirig blocks desta teoria com a utilização concreta do métoco.

Lucas (1981, p.217) define ciclos econômicos como movimentos de séries temporais em torno de uma tendência. Estes movimentos, no entanto, "...do not exhibit uniformity of either period or amplitude, which is to say, they do not resemble the deterministic wave motions which sometimes arise in the natural science". As regularidades manifestam-se na forma de co-movements comuns a todos os ciclos econômicos, entre os quais os mais importantes são:

i. Output movements across broadly defined sectors move together,

ii. Production of producer and consumer durables exhibits much greater amplitude than does the production of nondurables;

iii. Production and prices of agricultural goods and natural resources have lower than average conformity;

iv. Business profits show high conformity and much greater amplitude than other series;

v. Prices generally are proryclical;

vi. Short-term interest rates are procyclical; long-term rates s!:

vii. Monetary aggregates and velocity measures are procyciical.

Sargent (1979, p.256), por outro lado, oferece uma definição de ciclos que também enfatiza os co-movements:

The business cycle is the phenomenon of a number of important economic aggregates (such as GNP, unemployment and lay offs) being cnaracterized by high pairwise coherence at the low business cycle frequencies, the same frequencies at which most aggregates have most of their spectral power if they have 'typical spectral shapes'.

4 Note-se que relaçōes empíricas (correlaçōes), nesta acepção, podem ser confundidas com causalidade. Nāo obstante, neste caso, as relaçōes de causalidade - se corretamente definidas dependerảo de séries temporais relevantes (do período) e do uso preciso de técnicas econométricas. Este procedimento, contrariamente ao pretendido, não consegue eliminar o juízo de valor do observador ou de práticas arbitrárias. 
Vercelli (1991, p.133) corretamente pondera que a definição de ciclos de Lucas limita-se às regularidades invariantes, que fazem parte da noção de equilíbrio adotada, a qual requer que o processo estocástico seja estacionário. Na realidade, ao destacar os co-movements característicos dos ciclos, Lucas (1981, p.218) está sugerindo "...the possibility of a unified explanation of business cycles, grounded in the 'general' laws governing market economies, rather than in political or institutional characteristics specific to particular countries of periods". O objetivo dos Novos Clássicos era, portanto, desenvolver uma teoria de ciclos geral, que também fosse uma "teoria de equilíbrio competitivo". Logo, seria necessário justificar não apenas as vantagens que a adoção de uma "teoria de equilíbrio" traria, mas também de definir o escopo e limite do conceito de equilíbrio a ser usado.

Lucas e Sargent (1981) criticam Keynes por ter rompido com o rigor imposto pelos Clássicos. Esta disciplina impunha-se pela obrigatoriedade de atender a duas suposiçōes: mercados competitivos (asseguram a existência de fundamentos microeconômicos) e agentes que atuam sempre na defesa de seus próprios interesses (estabelece um padrão comportamental). Os supostos acima não poderiam ser reconciliados com a gravidade da depressão e com o desemprego generalizado (ibid., p.304). Para resolver este impasse, Keynes (1936) teria usado um método em que "...rules of thumb, such as the consumption function and liquidity preference schedule, took the place of decisions functions that a classical economist would insist be derived from the theory of choice. And rather than require that wages and prices be determined by the postulate that markets clear...Keynes took as an unexamined postulate that money wages are sticky, meaning that they are set at a level or by a process that could be taken as uninfluenced by the macroeconomic forces he proposed to arialyze", ou seja, Lucas e Sargent (1981) criticam Keynes por ter abusado de procedimentos arbitrários, ad $h o c$, sem respaldo empírico e pela ausência de fundamentos microeconômicos.

A passagem acima sugere que Keynes ao romper com o método clássico teria praticamente induzido a Ciência Econômica a um retrocesso fcrçado Tudo porque Keynes $^{5}$ teria abandonado o equilibrium method. A síntese neoclássica também não escapou às críticas de Lucas $(1981$, p.293) pelo fato de que "...the concept of market equilibrium used was that of static, deterministic general equilibrium theor y". A alternativa dos Novos Clássicos, naturalmente, seria a de introduzir no estudo dos ciclos econômicos um método que fosse "dinâmico, estoćástico e de equilíbrio competitivo", o qual representa, na realidade, o núcleo da

5 Observe aqui que Lucas e Sargent (1981, p.305) erroneamente concluiram que Keynes (1936) faz com que o termo equilfbrio "...was thought to refer to a systema at rest...", que serviria também para caracterizar a Teoria Neoclássica. Vercelli $(1991$, p.15) destaca, porém, que "There may be dynamic processes which...involve variables different from the state variable, whose influence on the latter adds up to zero through reciprocal compensation". Além disso, o conceito de equilibrio em Keynes (1936) é dinâmico. 
contra-revolução clássica. ${ }^{6} \mathrm{O}$ problema, portanto, seria solucionado ao definir-se a economia como sujeita a um processo estocástico multivariado, em conjunto com uma equilibrium theory, nos limites da teotia walrasiana,

A identificação de regularidades invariantes, ou co-movements de variáveis ao longo dos ciclos, constitue-se como estágio preparatório para a introdução do método de equilibrio proposto, o qual, por seu turno, sustenta-se na concepção de que a economia em equilíbrio obedece a um processo estocástico estacionário. A definição de séries temporais estacionárias é a que segue abaixo (Hannan, 1990 , p.271):

An infinite sequence $y(t), t=0,+i-1, \ldots$, of random variables is called stationary if the joint probability laws of $y\left(t_{1}\right), y\left(t_{2}\right), \ldots, y\left(t_{n}\right)$ is the same as that of $y\left(t_{1}+t\right), \ldots, y\left(t_{n}+t\right)$ for any integers, $\mathfrak{t}_{1}, t_{2}, \ldots$, $t_{0}, t$ and any $n$

Sob o ponto de vista estritamente estatístico, duas implicações centrais destacam-se. A mais importante conclui que o mecanismo estocástico que gera a seqüência acima é invariante. Além disso, a definição acima insere-se em um contexto ergódico. O significado econômico de séries temporais estacionárias, contudo, é mais complexo, notadamente quando aplicadas ao esțdo de ciclos econômicos. O próximo passo, portanto, será o de avaliar o significado do termo "equilibrio", como comumente usado pelos Novos Clássicos, além de investigar o significado de modelo ergódico na análise econômica.

Não é objetivo deste artigo discutir exaustivamente o significado de equilíbrio, quer do ponto de vista semântico ou filosofico. No entanto, como já discutido, a percepção de que o método de Keynes era "estático e determinístico" merece uma maior apreciação. Vercelli $(1991$, p.15) ressalta que o conceito de squilibrio de Lucas assenta-se em dois requisitos:

i. deve emergir como uma decisão ótima de parte dos agentes econômicos agindo em função de seus próprios interesses individuais; e

ii. não deve haver excesso de oferta ou demanda.

Como se sabe, o equilíbrio pode ser estático ou móvel (moving equilibrium, na terminologia de Keynes). A diferença entre estes dois tipos de equilíbrio refere-se à natureza do efeito das relações estruturais ao longo do tempo. Vercelli $(1991$, p.14) destaca que: "According to the dynamic concept, a system is in equilibrium whenever it is not charactirized by an endogenous dynamic process". Acontece, no entanto, que quando os dois requisitos acima são atendidos, eliminam-se as possibilidades de comportamentos dinâmicos endógenos.

6 Nāo é sensato supor-se que os Novos Clássicos tenham pretendido discutir e/ou redefinir princípios fundamentais. O esforço de originalidade e lógica concentrou-se na construçăo de um "novo" método, que fosse empiricamente robusto, no sentido de previsão (não de explicação), acoplado ao equilibrium theory. 
Este fato não exclui, contudo, a existência de equilíbrio movel, porquanto processos estocásticos compatíveis com choques aleatórios exógenos asseguram a validação do conceito de equilíbrio resultante de processos estocásticos estacionários. Em outras palavras, s6 choques exogenos podem provocar distúrbios na posição de equilíbrio.

Com o procedimento acima descrito, os Novos Clássicos conseguiram livrar-se de dois grandes problemas ao mesmo tempo. Não apenas puderam evitar as restrições impostas pela nocão restrita de equilíbrio ("nada muda no sistema"), isto $e$, a idéia de equilíbrio estático e estacionário, como também construíram uma teoria a partir do reconhecimento de que o comportamento da economia obedece a um processo estocástico e dinâmico. Em outras palavras, teriam resolvido duas antigas e indesejáveis características do método neoclássico: o equilíbrio estático e o determinismo. A nova proposta consistia, portanto, na adoção de processos estocásticos e equilíbrio móvel, a partir de choques exógenos, dentro dos limites do equilibrium method em modelos macroeconômicos, que estendessem o conceito de equilíbrio geral. Este fato, talvez, seja o que menor ilustre a ciggenhosidade do método dos Novos Clássicos, que não apenas moderniza a teoria neoclássica tradicional, mas até mesmo supera-a quanto ao grau de generalidade.

\section{EQUILÍBRIO E DINÂMICA: UMA AVALIAÇÃO CRÍTICA}

Parece não restar dúvidas que, sob o ponto de vista do método, os Novos Clássicos adotaram princípios que superam algumas dificuldades experimentadas peio neoclassismo histórico. Abstraindo-se da discussão pormenorizada de problemas teóricos, que não é o objetivo deste artigo, permanecem ainda várias questões a serem examinadas quanto às propriedades do método novo clássico.

Como discutido na seção 4, a Teoria dos Ciclos Econômicos dos Novos Clássicos pressupõe a identificação de regularidades sistemáticas a partir do estucio de séries temporais de variáveis relevantes. Este fato possui extraordinária importância, conquanto é base nas regularidades que a teoria de decisão opera. Ao mesmo tempo, os Novos Clássicos dispenderam energia e criatividade para construir um método fundamentado em processos estocásticos e dinâmicos, que fosse compatível com o equilibrium met'́od. É exatanıente por esta razão que

7 Os Novos Clássicos parecem reconhecer que modelos lineares determinísticos apresentam fases ascendentes e descendentes completamente simétricas. Blatt (1980) observou que a porção ascendente dos ciclos é mais longa e tem uma inclinação média menor que a porção descendente. Modelos estocásticos lineares, por outro lado, sāo menos simét:icos que modelos determinísticos, no en tan to, nāo deixam de ser simétricos, porquanton não exibem assimelria sistemática. Só modelos não-lineares - incompatíveis con o método dos Novos Clássicos - podem expressar com precisäo as assimetrias entre a fase ascendente e a descendente dos ciclos. Logo, a existência ou não de assimetrias também é um problema metodológico relevante.

8 A construção de modelos estocásticos e dinà micos é uma solução apenas parcial ao desafio proposto por Lucas (1981) de construir uma teoria de ciclos que possa ser expressa na forma de equilíbrio competitivo. $\mathrm{Na}$ realidade, a contradição persiste, pois este método assume que todos os estados de equilíbrio são eficientes. Isto significa dizer que tanto na recessāo qua nto nos booms a economia opera eficientemente, ou seja, não há reduçāo no bem-estar durante as recessōes. Aqueles, involuntariamente desempregados, certamente não concordam com esta conclusāo.

9 E importante relembrar que o conceito de regularidades insere-se como característica de sistemas ergodicos. 
surge uma clara contradição no método, como também concorda Vercelli (1991). $\mathrm{Na}$ realidade isto fica evidenciado pela constatação que o sistema econômico observável é visto como estável - marcado por regularidades - enquanto o modelo proposto é estruturalmente instável. Burmeister (1980, p.801) destaca que "Saddlepoint instability is a generic dynamic property of existing multi-market models featuring perfect foresight, competition, and portfolio equilibrium". A razão pela qual o equilíbrio dá-se no ponto de cela é que (ibid., p.803-804):"If all sthocastic shocks were in fact identically zero, these expectations paths would be identical to the perfect foresight paths...consequently it is not surprising that a dynamic system of rational expectations path is often featured by a saddlepoint equilibrium". Burmeister (ibid., p.805) conclui afirmando que "One is left with the most unconfortable conclusion that in some (Many? Most?) rational expectations models not having convergent expectations, the sthocastic behavior of the actual variables is indeterminate" ${ }^{10}$ Isto significa dizer que se a realidade não for estável - se os co-movements não ocorrerem de forma repetitiva - qualquer pequeno desvio do modelo em relação à realidade observada tornará a previsão do comportamento futuro da economia impossível. Isto é incompatível com a hipotese das expectativas racionais. De fato, a lógica e estratégia do método novo clássico aqui são expressas de forma criativa, pois o modelo ao ser estocástico e dinâmico, elimina críticas normalmente associadas aos modelos neoclássicos tradicionais que seriam estáticos e determinísticos - mantendo-se fiel ainda ao equilihrium method. Este resultado surpreendente só é obtido, no entanto, porque o modelo dos Novos Clássicos é aplicado sobre uma realidade estacionária e repetitiva.

O objetivo central da crítica, ora a ser desenvolvida, centra-se nas condições e mecanismos necessários para que as regularidades invariantes - ponto central do método novo clássico - possam projetar-se ao longo dos ciclos econômicos, tornando, então, o resultado de processos decisórios uma simples questão de eswolher o método estatístico correto.

O ponto de partida para a identificação das regularidades invariantes relaciona-se como o estabelecimento de regras comportamentais seguidas pelos agentes econômicos. Aqui os agentes são "inteligentes", isto é, são capazes de "corrigir" seus erros. Os agentes aprendem atraves de uma função de probabilidade subjetiva, que na realidade é a função "verdadeira". Lucas (1981, p.223), descrevendo o artigo de Muth (1961), destaca que este propôs com sucesso um procedimento que permite entender como o processo de decisão dos agentes $\epsilon$ empreendido:"...by identifying agents' subjective probabilities with observed frequencies of the event to be forecast, or with 'true' probabilities, calling the assumed coincidences of subjective and 'true' probability rational expectations".

10 Para tentar evitar boa parte destes problemas, os Novos Clássicos usam - sem parcimônia - os chamados "free parameters", ou parâmetros de ajustamentos para garantir a convergência das expectativas. Logo, nảo é difícil entender a elevada dose de arbitrariedade envolvida nesse tipo de procedimento. 
E acrescenta, "Evidently, this hypothesis will not be of value in understanding psychotic behavior". Por comportamento psicótico deve-se entender qualquer ação dos agentes que não tenha um caráter otimizador, uma vez que os mesmos possuem pleno conhecimento da distribuição "verdadeira". Além disso, é assumido que os agentes decidem com base em preferências e tecnologia constantes (Lucas, 1981, p.220-221). Lucas (ibid., p.224) conclui afirmando que: "In cases of uncertainty, economic reasoning will be of no value".

As considerações acima ilustram a maneira como os Novos Clássicos decretam a irrelevância das incertezas nas decisốcs econômicas. ${ }^{11}$ A razão disso, como sugere Lawson $\left(1988\right.$, p.59) e que ao se introduzir o conceito de incerteza ${ }^{12}$ "...there will be no obvious recurrent event on the basis of which economistis may calibrate some supposed agent subjective distribution using the rational expectations approach". Esta conclusão é corroborada por Lucas (1981, p.224), quando afirma que:

Insofar as business cycles can be viewed as repeated instances of essentially similar events, it will be reasonable to treat agents as reacting to cyclical changes as 'risk', or to assume their expectations are rational, that they have fairly stable arrangements for collecting and processing information, and that they utilize this information in forecasting the future in a stable way, free systematic and easily and correctable biases.

Pode-se, então, derivar dois importantes corolários a partir da citação acima e de acordo com a conclusão de Lawson, que reduzem significativamente o alcance do método dos Novos Clássicos. Em primeiro lugar, se é verdade que o processo de decisão do qual os agentes fazem parte - como comentado acima - é estocástico, neste método a realidade é estacionária. Não obstante isto, o processo repete-se o bastante para que os agentes aprendam e ajustem-se perfeitamente, sem erros cumulativos. Ou ainda, o processo de decisão situa-se em um cenário de relações ergódicas, reflexo de uma realidade tida como estruturalmente estável, inclusive nas preferências e tecnologia ao longo dos ciclos econômicos. Neste modelo, não há espaço para "surpresas potenriais".

O grande problema resultante da irrelevância das incertc $\iota$ as manifesta-se no papel desempenhado pela variável tempo e, logo, no conceito de equilíbrio e em relação ds surpresas potenciais. No primeiro caso, os Novos Clássicos associam-se a realidade com uma série de eventos similares correlacionados entre si, donde surge a completa alienação do tempo histórico, ou ainda, das condições iniciais como variáveis críticas no processo econômico. Por outro lado, em um cenário caracterizado por incertezas, não parece núm um pouco provável esperar-se que a função de probabilidade subjetiva dos agentes coincidam com a função "verdadeira". Assim, é perfeitamente razoável supor-se que os agentes possam cometer erros sistemáticos. Estes estão longe de significarem algum tipo de

11 No sentido de Keynes, como exposto em Lawson (1988)

12 Observe-se que os Novos Clássicos não reduzem as incertezas ao mesmo status de risco, como comum a boa parte da literatura neoclássica con temporânea. No caso em questāo, as incertezas são sumariamente eliminadas da análice. 
"comportamento psicótico", como sugere Lucas. Tampouco significa que os agentes sejam irracionais. Significa apenas que as relaçóes econômicas são volateis. O momento histórico em que as decisões são tomadas é irreproduzível, único. Is to equivale a dizer que, a menos que os agentes econômicos possuam um poder de premonição sobrenatural - como as cartomantes -, suas funções de probabilidade subjetiva nunca serão idênticas à distribuição "verdadeira".

\section{CONCLUSÃO}

O esforço central dos Novos Clássicos - pouco ou nada têm em comum com os Clássicos - foi o de produzir um método que subordinasse a macroeconomia keynesiana à lógica axiomática da ortodoxia walrasiana. Neste sentido, alinham-se a um tipo de instrumentalismo sofisticado, onde os ciclos econômicos são definidos como sendo eventos repetitivos, caracterizados por co-movements entre as variáveis relevantes. A partir daí, introduzem o conceito de equilíbrio estacionário, com a novidade de adotarem um modelo estocástico compatível com processos dinâmicos e choques exógenos, ou seja, eliminam formalmente alguns problemas típicos do método neoclássico trádicional, classificado como estático e determinístico, que é substituído por outro, dinâmico e estocástico, mantendo, contudo, o mesmo princípio organizador comum dos Neoclássicos, isto é, o equilibrium method.

A engenhosidade do método novo clássico decorre da adoção de um método que é tecnicamente dinâmico, mas que so consegue projetar-se sobre uma realidade marcada por regularidades, ou seja estacionária. Se não for este o caso, o modelo torna-se dinamicamente instável. Em outras palavras, torna-se inapto para fazer previsões se a realidade não for estacionária.

Em suma, a sofisticação do método novo clássico não conseguiu, de fato, reduzir a enorme complexidade das relações mıacroeconômicas ao dom ínio estreito do equilíbrio wairasiano, conquanto é, impossível conciliar tempo e incerteza com o equilibrium method. Nenhum modelo econômico pode escapar de algum elemıcnto de arbitrariedade. Em relação aos Novos Clássicos, parece ter havido um notável exagero.

\section{BIBLIOGRAFIA}

BLATT, J. On the Frisch Model of Business Cycles OxfordEconcmic Papers, v.32, p.467-469, 1980. BURMEISTER, E. On Some Conceptual Issues in Rational Expectations Modeling. Journal of Money, Credit, and Banking, v.12, n.2, p. Sü0-816, 1980.

FRIEDMAN, M. Essays in Positive Economics. Chicago: University of Chicago Press, 1953.

HANNAN, F. Stationary Time Series. In: EATWELL, I et alii (orgs.) The New Palgrave: a Dicionary of Economics. New York: Macmillan, 1990.

HICKS, J. IS-LM: an Explanation, Journal of Post Keynesian Economics, v.3, p. 139-54, 1980-81. ...- Methods of Dynamic Economics. Oxford: Clarendon Press, 1985.

KEYNES, J. M. The Gencral Theory of Employment, Interest and Money. London: Macmillan, 1936. L.AWSON, T. Probability and Uncertainty in Economic Analysis. Journal of Post Keynesian

Economics, v.11, p.38-65, 1988.

LUCAS, R. Studies in Business-Cycle Theory. Cambridge: MIT Press, 1981. 
-.-.. Models of Business Cycles. New York: Basil Blackwell, 1987.

-..- e RAPPING, L. Real Wages, Employment and Inflation. Journal of Political Economy, v.77, p.721-54, 1969.

---- SARGENT, T. Rational Expectations and Econometric Practice. Minneapolis: University of Minnesota Press, 1981.

MUTH, J. Rational Expectations and the Theory of Price Movements. Econometrica, v 29, p.315-35. 1961.

SARGENT, T. Macroeconomic Theory. New York: Academic Press, 1979

VERCELLI, A Methodological Foundations of Macroecononics: Keynes \& Lucas. Cambridge: Cambridge University Press, 1991.

\section{ABSTRACT \\ THE NEW CLASSICALS ANJ THE METHOD}

In the beginning of the 1970's, the New Classical School challenged the foundations of keynesianism, based on the lack of microfoundations of the General Theory. The objective of the New Classical School was to construct a business cycle theory compatible with the competitive equilibrium, as an alternative to keynesian economics. The identification of regularities allowed a unified explanation for business cycles. This method, however, assumes that the actual reality is stationary. If that is not the case the model becomes dynamically unstable. 